\title{
Mean-field theory for symmetry-breaking Fermi surface deformations on a square lattice
}

\author{
Hiroyuki Yamase ${ }^{1}$, Vadim Oganesyan ${ }^{2}$, and Walter Metzner $^{1}$ \\ ${ }^{1}$ Max-Planck-Institute for Solid State Research, \\ Heisenbergstrasse 1, D-70569 Stuttgart, Germany, \\ ${ }^{2}$ Department of Physics, Princeton University, Princeton, NJ 08544, USA
}

(Dated: May 23, 2022)

\begin{abstract}
We analyze a mean-field model of electrons with pure forward scattering interactions on a square lattice which exhibits spontaneous Fermi surface symmetry breaking with a $d$-wave order parameter: the surface expands along the $k_{x}$-axis and shrinks along the $k_{y}$-axis (or vice versa). The symmetry-broken phase is stabilized below a dome-shaped transition line $T_{c}(\mu)$, with a maximal $T_{c}$ near van Hove filling. The phase transition is usually first order at the edges of the transition line, and always second order around its center. The $d$-wave compressibility of the Fermi surface is however strongly enhanced even near the first order transition down to zero temperature. In the weak coupling limit the phase diagram is fully determined by a single non-universal energy scale, and hence dimensionless ratios of different characteristic quantities are universal. Adding a uniform repulsion to the forward scattering interaction, the two tricritical points at the ends of the second order transition line are shifted to lower temperatures. For a particularly favorable choice of hopping and interaction parameters one of the first order edges is replaced completely by a second order transition line, leading to a quantum critical point.

PACS numbers: 71.18.+y, 71.10.Fd
\end{abstract}

\section{INTRODUCTION}

The low energy properties of an interacting electron system are strongly influenced by the shape of its Fermi surface. Residual interactions between quasi-particles near the Fermi surface can give rise to charge- or spin-density waves, superconductivity, or other low energy instabilities. Usually the Fermi surface respects the point-group symmetry of the underlying 
lattice structure. In principle, however, electron-electron interactions can drive a Fermi surface deformation that breaks the orientational symmetry of the system.

Recently, the possibility of symmetry-breaking Fermi surface deformations with a $d$-wave order parameter, where the surface expands along the $k_{x}$-axis and shrinks along the $k_{y}$-axis (or vice versa), was discussed for various two-dimensional electron models on a square lattice: $t-J, \frac{1}{,}$ Hubbard $\stackrel{2.3 .4}{\underline{4}}$ and extended Hubbard $\mathrm{f}^{\underline{5}}$ model. The instability is driven by interactions in the forward scattering channel, mainly between electrons close to the van Hove points in the two-dimensional Brillouin zone. Referring to Pomeranchuk's $s^{\underline{6}}$ stability condition for isotropic Fermi liquids it has been termed "Pomeranchuk instability" by some authors. Only a discrete lattice symmetry is broken by the $d$-wave deformation on the square lattice, in constrast to spontaneous Fermi surface symmetry breaking in isotropic Fermi liquids, where Goldstone modes play an important role.$^{\underline{T}}$ The $d$-wave Fermi surface deformation leads to a state with the same reduced symmetry as the "nematic" electron liquid defined by Kivelson et al $\underline{\underline{8}}$ in a discussion of possible analogies between doped Mott insulators with charge stripe correlations and liquid crystal phases. $\underline{\underline{9}}$

Spontaneous Fermi surface symmetry breaking competes with other instabilities. In the slave-boson mean-field theory of the $t$ - $J$ model the $d$-wave Fermi surface deformation is overwhelmed by $d$-wave singlet pairing, but strongly enhanced correlations in the $d$-wave forward scattering channel and a corresponding large response to external anisotropic perturbations remain. $\stackrel{1.10}{=}$ The latter can be related to distinctive properties of magnetic excitations in different cuprate superconductors: ${ }^{1.11}$ In the Hubbard model near van Hove filling coexistence of superconductivity and $d$-wave Fermi surface symmetry breaking has been found in a renormalized weak coupling perturbation expansion. $\underline{\underline{4}}$

Quantum critical fluctuations of the "soft" Fermi surface near a continuous zero temperature phase transition with Fermi surface symmetry breaking provide a route to non-Fermi liquid behavior. $\frac{12}{2}$ Anomalously large and anisotropic quasi-particle decay rates have been derived for a phenomenological model, where electrons moving on a square lattice interact only via almost forward scattering interactions, that is only very small momentum transfers are allowed $\stackrel{\underline{\underline{12}}}{\underline{*}}$ We refer to this model as the "f-model" in the following. Recently it was shown that the putative quantum critical point in the f-model is actually preempted by a first order transition, at least within mean-field theory and for various concrete choices of the model parameters $\stackrel{13}{\underline{13}}$ The transition remains first order at low finite temperatures but 
turns to second order at temperatures above a tricriticial point $\underline{\underline{14}}$

This paper is dedicated to a comprehensive mean-field analysis of spontaneous Fermi surface symmetry breaking in the f-model on a square lattice. We present results for the phase diagram, order parameter and Fermi surface as obtained from a numerical solution of the mean-field equations for various typical choices of parameters. The weak coupling limit is analyzed analytically. We also compute the coefficients of the Landau expansion of the grand canonical potential up to quartic order in the order parameter and show that the first order transition at low temperature is a rather robust consequence of the van Hove singularity in the density of states. Besides confirming the conclusions by Kee et al $\stackrel{13}{\underline{13}}$ and Khavkine et al. .14 $^{4}$ and providing additional numerical data especially at finite temperatures, we present several new results and insights. In particular, we show that in the weak coupling limit Fermi surface symmetry breaking is characterized by a single energy scale, which leads to universal behavior in terms of suitably rescaled parameters. Furthermore, we show that the tricritical points can be suppressed to lower temperatures by a uniform repulsion added to the original f-model, which, for a particularly favorable but not unphysical choice of hopping and interaction parameters, can even lead to a quantum critical point. Finally, we find that the $d$-wave compressibility of the Fermi surface is usually strongly enhanced along the transition line down to zero temperature even if the transition is first order, which implies that the Fermi surface is already very soft at the transition and fluctuations should be important.

The article is structured as follows. In Sec. II we define the f-model and outline the meanfield theory of spontaneous Fermi surface symmetry breaking. Results from a numerical solution of the mean-field equations are presented in Sec. III. The numerical results are complemented by an analysis of the Landau free energy expansion in Sec. IV and an analytic derivation of universal properties of the phase transition at weak coupling in Sec. V. We finally conclude in Sec. VI. 


\section{MODEL AND MEAN-FIELD THEORY}

We analyze the f-model on a square lattice with pure forward scattering interactions. The Hamiltonian reads

$$
H=\sum_{\mathbf{k}} \epsilon_{\mathbf{k}}^{0} n_{\mathbf{k}}+\frac{1}{2 L} \sum_{\mathbf{k}, \mathbf{k}^{\prime}} f_{\mathbf{k k}^{\prime}} n_{\mathbf{k}} n_{\mathbf{k}^{\prime}}
$$

in standard second quantized notation, where $n_{\mathbf{k}}=\sum_{\sigma} n_{\mathbf{k} \sigma}$ counts the spin-summed number of electrons with momentum $\mathbf{k}$, and $L$ is the number of lattice sites. For hopping amplitudes $t, t^{\prime}$, and $t^{\prime \prime}$ between nearest, next-nearest, and third-nearest neighbors on the square lattice, respectively, the bare dispersion relation is given by

$$
\epsilon_{\mathbf{k}}^{0}=-2\left[t\left(\cos k_{x}+\cos k_{y}\right)+2 t^{\prime} \cos k_{x} \cos k_{y}+t^{\prime \prime}\left(\cos 2 k_{x}+\cos 2 k_{y}\right)\right] .
$$

The forward scattering interaction has the form

$$
f_{\mathbf{k k}^{\prime}}=u-g d_{\mathbf{k}} d_{\mathbf{k}^{\prime}}
$$

with coupling constants $u \geq 0$ and $g>0$, and a function $d_{\mathbf{k}}$ with $d_{x^{2}-y^{2}}$-wave symmetry such as $d_{\mathbf{k}}=\cos k_{x}-\cos k_{y}$. This ansatz mimics the structure of the effective interaction in the forward scattering channel as obtained for the $t-J, \underline{1}$ Hubbard, $\stackrel{2}{\stackrel{2}{p}}$ and extended Hubbard $\stackrel{5}{\underline{5}}$ model. The uniform term originates directly from the repulsion between electrons and suppresses the (uniform) electronic compressibility of the system. The $d$-wave term enhances the $d$-wave compressibility of the Fermi surface and drives spontaneous Fermi surface symmetry breaking. In the Hubbard model it is generated by (1-loop) fluctuations, while in the $t-J$ and extended Hubbard model the nearest neighbor interaction contributes directly to a $d$-wave attraction in the forward scattering channel. For $u=0$, the above model is the pure forward scattering limit of the f-model with small momentum transfers introduced in Ref. 12. Within mean-field theory, it is also equivalent to the model analyzed in Refs. 13, 14, since the off-diagonal components of the quadrupole density introduced there do not affect the results.

Inserting $n_{\mathbf{k}}=\left\langle n_{\mathbf{k}}\right\rangle+\delta n_{\mathbf{k}}$ into the interacting part of the model and neglecting terms of order $\left(\delta n_{\mathbf{k}}\right)^{2}$, we obtain the mean-field Hamiltonian

$$
H_{\mathrm{MF}}=\sum_{\mathbf{k}} \epsilon_{\mathbf{k}} n_{\mathbf{k}}-\frac{1}{2} \sum_{\mathbf{k}} \delta \epsilon_{\mathbf{k}}\left\langle n_{\mathbf{k}}\right\rangle
$$


where $\epsilon_{\mathbf{k}}=\epsilon_{\mathbf{k}}^{0}+\delta \epsilon_{\mathbf{k}}$ is a renormalized dispersion relation, which is shifted with respect to the bare dispersion by

$$
\delta \epsilon_{\mathbf{k}}=\frac{1}{L} \sum_{\mathbf{k}^{\prime}} f_{\mathbf{k k}^{\prime}}\left\langle n_{\mathbf{k}^{\prime}}\right\rangle=u n+\eta d_{\mathbf{k}} .
$$

Here $n=L^{-1} \sum_{\mathbf{k}}\left\langle n_{\mathbf{k}}\right\rangle$ is the average particle density, and

$$
\eta=-\frac{g}{L} \sum_{\mathbf{k}} d_{\mathbf{k}}\left\langle n_{\mathbf{k}}\right\rangle
$$

is our order parameter, which parametrizes the amount of symmetry breaking. Note that $\eta$ is real and has the dimension of energy. It vanishes as long as the momentum distribution function $\left\langle n_{\mathbf{k}}\right\rangle$ respects the symmetry of the square lattice. The grand canonical potential per lattice site $\omega=L^{-1} \Omega$ is obtained from the mean-field Hamiltonian as

$$
\omega=\frac{\eta^{2}}{2 g}-\frac{u}{2} n^{2}-\frac{2 T}{L} \sum_{\mathbf{k}} \log \left(1+e^{-\left(\epsilon_{\mathbf{k}}-\mu\right) / T}\right) .
$$

The stationarity conditions $\frac{\partial \omega}{\partial \eta}=0$ and $\frac{\partial \omega}{\partial n}=0$ (at fixed $\mu$ ) yield the self-consistency equation for the order parameter

$$
\eta=-\frac{2 g}{L} \sum_{\mathbf{k}} d_{\mathbf{k}} f\left(\epsilon_{\mathbf{k}}-\mu\right)
$$

and the equation determining the density

$$
n=\frac{2}{L} \sum_{\mathbf{k}} f\left(\epsilon_{\mathbf{k}}-\mu\right)
$$

respectively, where $f(\xi)=\left(e^{\xi / T}+1\right)^{-1}$ is the Fermi function. These equations follow also directly from the relation $\left\langle n_{\mathbf{k}}\right\rangle=2 f\left(\epsilon_{\mathbf{k}}-\mu\right)$. Note that the Eqs. (8) and (9) are coupled for $u \neq 0$, since $n$ enters the self-consistency equation for $\eta$ via $\epsilon_{\mathbf{k}}$.

In the thermodynamic limit the mean-field theory solves the reduced version (no momentum transfers) of the f-model exactly. One simple way to see this is by considering the Feynman diagrams representing the perturbation expansion of the system. All self-energy diagrams except the Hartree term involve integrals over momentum transfers and are thus suppressed at least by a factor $L^{-1}$.

\section{NUMERICAL RESULTS}

We first take band parameters $t^{\prime} / t=-1 / 6$ and $t^{\prime \prime}=0$, and solve the self-consistency equations eqs. (8) and (9) numerically. For this choice of hopping amplitudes the bare 
dispersion relation has saddle points at $(\pi, 0)$ and $(0, \pi)$, leading to a van Hove singularity in the bare density of states at $\epsilon_{\mathrm{vH}}^{0}=4 t^{\prime}=-2 t / 3$. Typical features of spontaneous Fermi surface symmetry breaking are captured with these parameters and are presented in the first three subsections. In the last subsection we investigate another set of hopping parameters, for which the saddle points are slightly shifted from $(\pi, 0)$ and $(0, \pi)$, and a quantum critical point can be realized for suitable choices of the interaction parameters.

\section{A. Typical phase diagram}

We first focus on the $d$-wave term in Eq. (33) and set $u=0$. A $\mu$-T phase diagram is shown in Fig. 1(a). The solid line denotes a second order phase transition, which turns to a first order transition at low $T$ (open circles). The end points of the second order transition are tricritical points (solid circles), where the quadratic and quartic coefficients of the Landau energy expansion (see Sec. IV) vanish simultaneously. The dashed line denotes the fictitious second order transition that is preempted by the first order transition. The complete transition line, $T_{c}(\mu)$, has a dome shape. Since the Fermi surface symmetry breaking is driven by forward scattering of electrons mainly on the (original) Fermi surface close to the van Hove points, $\stackrel{1.2}{,}$ the maximal $T_{c}(\mu)$ appears for $\mu$ around the van Hove energy $\epsilon_{\mathrm{vH}}^{0}=-2 t / 3$; a slight deviation from $\epsilon_{\mathrm{vH}}^{0}$ is due to finite $T$ effects. The transition line is almost symmetric with respect to the $\mu=\epsilon_{\mathrm{vH}}^{0}$ axis. This symmetry becomes exact when $t^{\prime}$ is set to zero because of particle-hole symmetry. Below $T_{c}$ the Fermi surface expands along the $k_{x}$-direction and shrinks along the $k_{y}$-direction or vice versa. We show results for the Fermi surface at low $T$ in Figs. 1(b) and (c) together with the corresponding bare Fermi surface $(g=0)$ for comparison. The Fermi surface has typically open topology in the symmetry-broken phase, except close to the second order transition.

In Fig. 1(d), we plot the order parameter $|\eta|$ as a function of $\mu$ at $T=0.01 t$ and $0.15 t$. While we see a continuous transition at $T=0.15 t,|\eta|$ exhibits a jump at $T=0.01 t$, characteristic of a first order transition. In Fig. 1(e), the density $n$ is plotted as a function of $\mu$ at $T=0.01 t$ and $0.15 t$ together with that for $g=0$. The density increases monotonuously with $\mu$. This behavior is due to the stability condition of the system that the grand canonical potential must be a concave function as a function of $\mu$, which yields an inequality, $-\frac{\partial^{2} \omega}{\partial^{2} \mu}>0$, or $\frac{\partial n}{\partial \mu}>0$. The density changes discontinuously at the first order transitions. The directions 

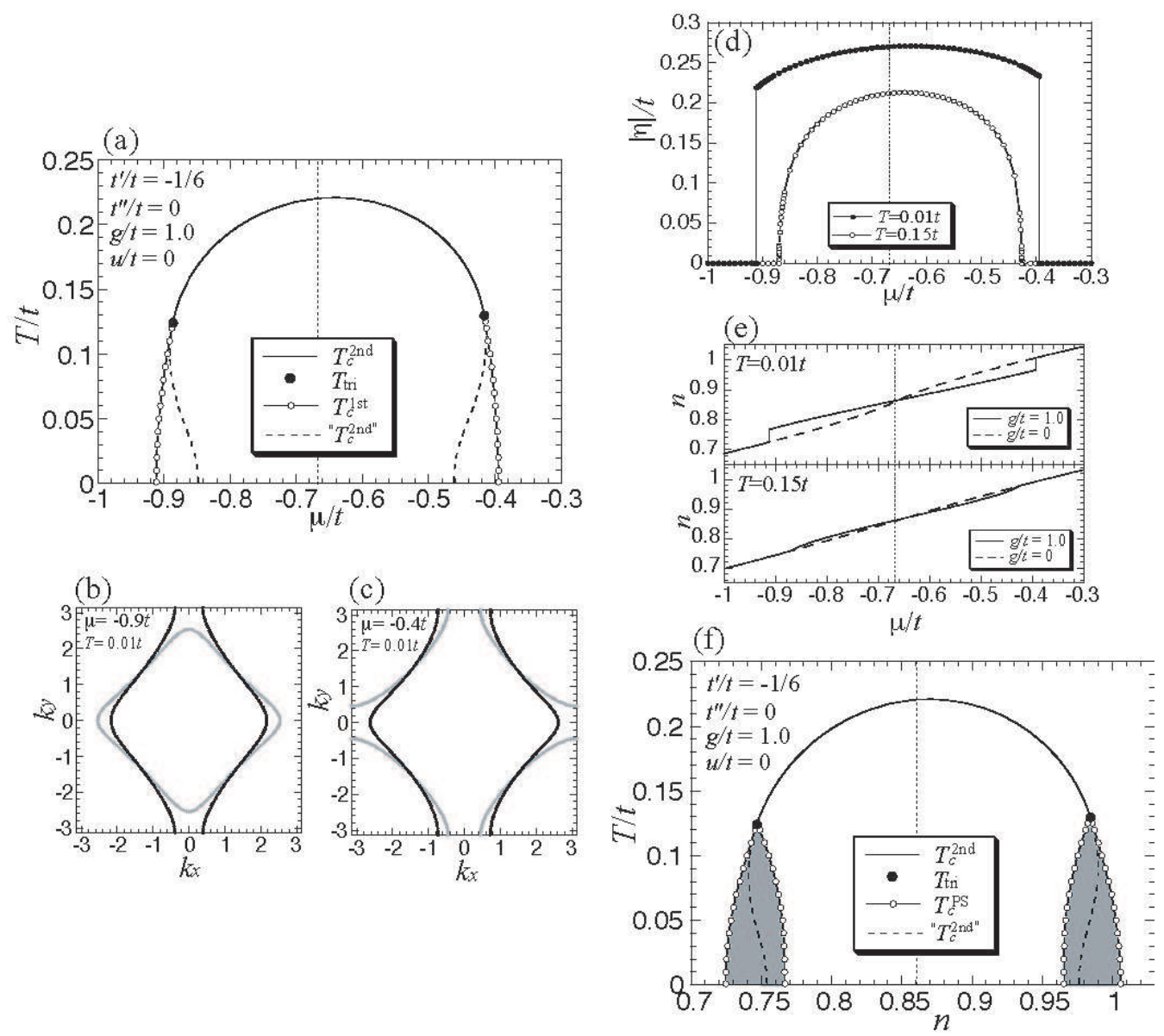

FIG. 1: The mean-field solution for $t^{\prime} / t=-1 / 6, t^{\prime \prime} / t=0, g / t=1$, and $u=0$. (a) $\mu$-T phase diagram; the total transition line, $T_{c}(\mu)$, contains a second order transition line, $T_{c}^{2 \text { nd }}$, at high temperatures and two first order lines, $T_{c}^{1 \text { st }}$, at low temperatures; the solid circles are tricritical points; the dashed line, " $T_{c}^{2 n d ", ~ d e n o t e s ~ a ~ f i c t i t i o u s ~ s e c o n d ~ o r d e r ~ t r a n s i t i o n ~ t h a t ~ i s ~ p r e e m p t e d ~ b y ~}$ the first order transition; the dotted line indicates the van Hove energy, $\mu=\epsilon_{\mathrm{vH}}^{0}=-2 t / 3$. (b) and (c) Fermi surface in the symmetry-broken phase near the first order transition; the Fermi surface for $g=0$ is also shown by a gray line. (d) $\mu$ dependence of $|\eta|$ at $T=0.01 t$ and $0.15 t$. (e) $\mu$ dependence of $n$ at $T=0.01 t$ and $0.15 t$; the results for $g=0$ are plotted also. (f) $n$ - $T$ phase diagram; $T_{c}^{2 \text { nd }}$ is a second order transition temperature and solid circles are tricritical points. In the shaded regions, which are surrounded by $T_{c}^{\mathrm{PS}}$, the system undergoes phase separation.

of the density jumps are generic features required by the concavity of the grand canonical potential.

In Fig. 1(f) the phase diagram is plotted in the $n-T$ plane. The second order transition 

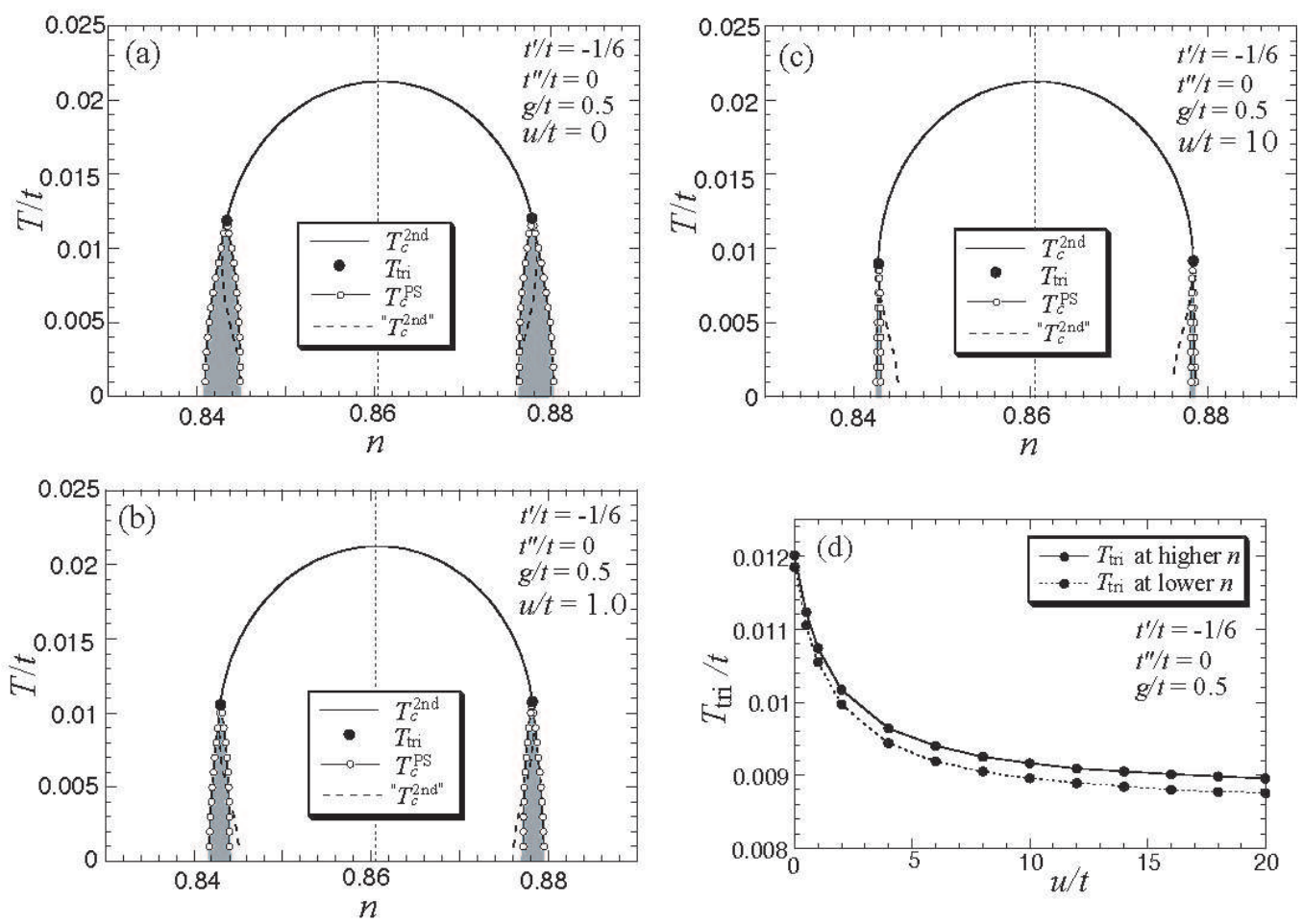

FIG. 2: $n$-T phase diagram for $t^{\prime} / t=-1 / 6, t^{\prime \prime} / t=0$, and $g / t=0.5$ with (a) $u=0$, (b) $u / t=1.0$, and (c) $u / t=10$. (d) $u$-dependence of $T_{\text {tri }}$ at higher $n$ (solid line) and at lower $n$ (dotted line).

line at high $T$ terminates at two tricritical points, below which phase separated regions, shown by shades, appear; dashed lines correspond to the ficticious second order transition shown already in Fig. 1(a). Each shaded region has two phases, with different densities $n_{1}$ and $n_{2}\left(>n_{1}\right)$. The difference between $n_{1}$ and $n_{2}$ corresponds to the magnitude of the density jump at the first order transition point [see Fig. 1(e)]. For $n_{1} \leq n \leq n_{2}$ the volume fraction of the low density phase and the high density phase is $\frac{n_{2}-n}{n_{2}-n_{1}}$ and $\frac{n-n_{1}}{n_{2}-n_{1}}$, respectively. The former has a symmetric (symmetry-broken) Fermi surface and the latter a symmetry-broken (symmetric) Fermi surface in the left (right) phase separated region.

\section{B. Effects of uniform repulsion}

Now we switch on the uniform term in the interaction, Eq. (3). The $n$ - $T$ phase diagrams are shown in Figs. 2(a)-(c) for several choices of $u$ at $g=0.5 t$. Note that typical features of the phase diagrams are the same as in Fig. 1 although we take smaller $g$ and finite $u$ here. The second order transition line is not affected by $u$ in the $n-T$ phase diagram, while it 
would be affected if plotted in the $\mu-T$ plane, because the chemical potential corresponding to a given density varies with $u$. The tricritical points, that is the end points of the second order transition, extend to lower $T$ with $u$ in favor of a second order transition, which is accompanied by a pronounced suppression of the width of the phase separated regions, since $n_{2}-n_{1}$ is strongly reduced by the uniform term in the interaction. However, we see that the suppression of $T_{\text {tri }}$ saturates at large $u$, as shown in Fig. 2(d), and is not strong enough to establish a quantum critical point.

\section{C. $d$-wave compressibility near the first order transition}

When the symmetry-broken phase is realized through a first order transition at low $T$, order parameter fluctuations are not critical at the transition. However, we now show that the anisotropic compressibility with a $d$-wave form factor is strongly enhanced by interactions at the transition line, such that fluctuations can be expected to be important in spite of the first order character of the transition.

The $d$-wave compressibility

$$
\kappa_{d}=\frac{d n_{d}}{d \mu_{d}}
$$

describes the linear response of the expectation value $n_{d}=L^{-1} \sum_{\mathbf{k}} d_{\mathbf{k}}\left\langle n_{\mathbf{k}}\right\rangle$ to the symmetrybreaking perturbation $H_{d}=-\mu_{d} \sum_{\mathbf{k}} d_{\mathbf{k}} n_{\mathbf{k}}$. The perturbation $H_{d}$ induces a $d$-wave shaped deformation of the Fermi surface. Note that the order parameter $\eta$ is directly proportional to $n_{d}$, namely $\eta=-g n_{d}$. Without the form factors $d_{\mathbf{k}}$ the above expressions would yield the

conventional compressibility $\kappa=\frac{d n}{d \mu}$. For our mean field model, the $d$-wave compressibility is given exactly by the RPA expression

$$
\kappa_{d}=\frac{N_{2}}{1-g N_{2}},
$$

where $N_{2}$ is a weighted density of states with $d_{\mathbf{k}}^{2}$ as a weight factor [see Eq. (16) in Sec. IV]. The denominator

$$
S^{-1}=1-g N_{2}
$$

is the inverse "Stoner factor", which is a dimensionless measure for the enhancement of the $d$-wave compressibility by interactions, and hence for the enhancement of order parameter fluctuations. 


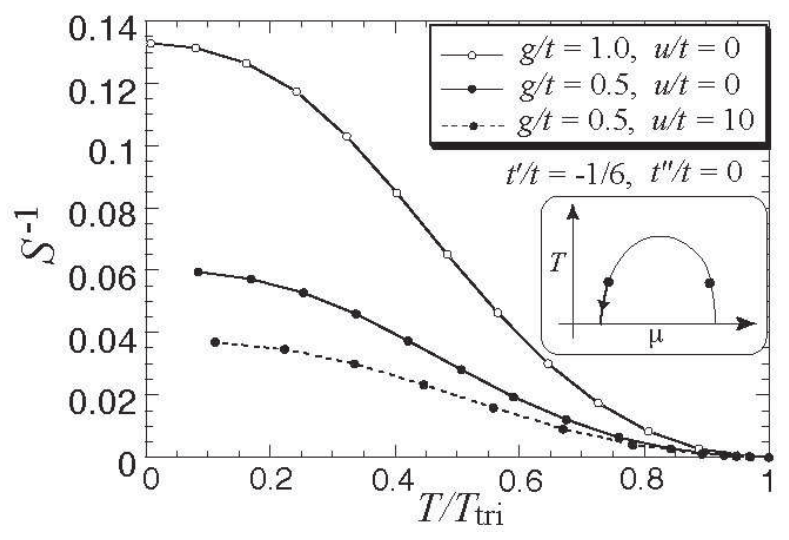

FIG. 3: The inverse of the Stoner enhancement factor for several choices of $g$ and $u$ along a first order transition line as sketched in the inset with the arrow; temperature is scaled by the tricritical temperature.

We calculate $S^{-1}$ along one of the two first order transition lines as sketched in the inset of Fig. 3. similar results are obtained along the other side of the first order transition. The main panel of Fig. 3 shows that $S^{-1}$ tends to zero at the tricritical temperature, that is the compressibility $\kappa_{d}$ diverges as expected, indicating truely critical fluctuations. At lower temperatures, $S^{-1}$ is finite on the transition line. However, its value is still much smaller than one, especially for a smaller $g$; the introduction of $u$ reinforces this tendency. The $d$-wave compressibility is thus strongly enhanced by interactions at the first order transition line down to the lowest temperatures, for example by a factor of about 25 for $g=0.5 t$ and $u=10 t$. Hence, Fermi surface and thus order parameter fluctuations can be expected to be important even near the first order transition at low $T$. In the weak coupling analysis presented in Sec. $\mathrm{V}$ we will show that $S^{-1}$ can be arbitrarily small for small $g$ near the first order transition.

\section{Quantum critical point}

The above results for our choice of hopping parameters, $t^{\prime} / t=-1 / 6$ and $t^{\prime \prime} / t=0$, are probably rather generic in the sense that other choices lead to qualitatively the same phase diagrams, as long as the bare kinetic energy has saddle points at $(\pi, 0)$ and $(0, \pi)$. Another interesting set of parameters is $t^{\prime} / t=-1 / 6$ and $t^{\prime \prime} / t=1 / 5$. For $t^{\prime \prime}>\frac{1}{4}\left(t+2 t^{\prime}\right)$ the bare kinetic energy has saddle points at $\left(\pi \pm \cos ^{-1} \alpha, 0\right)$ and $\left(0, \pi \pm \cos ^{-1} \alpha\right)$ with 

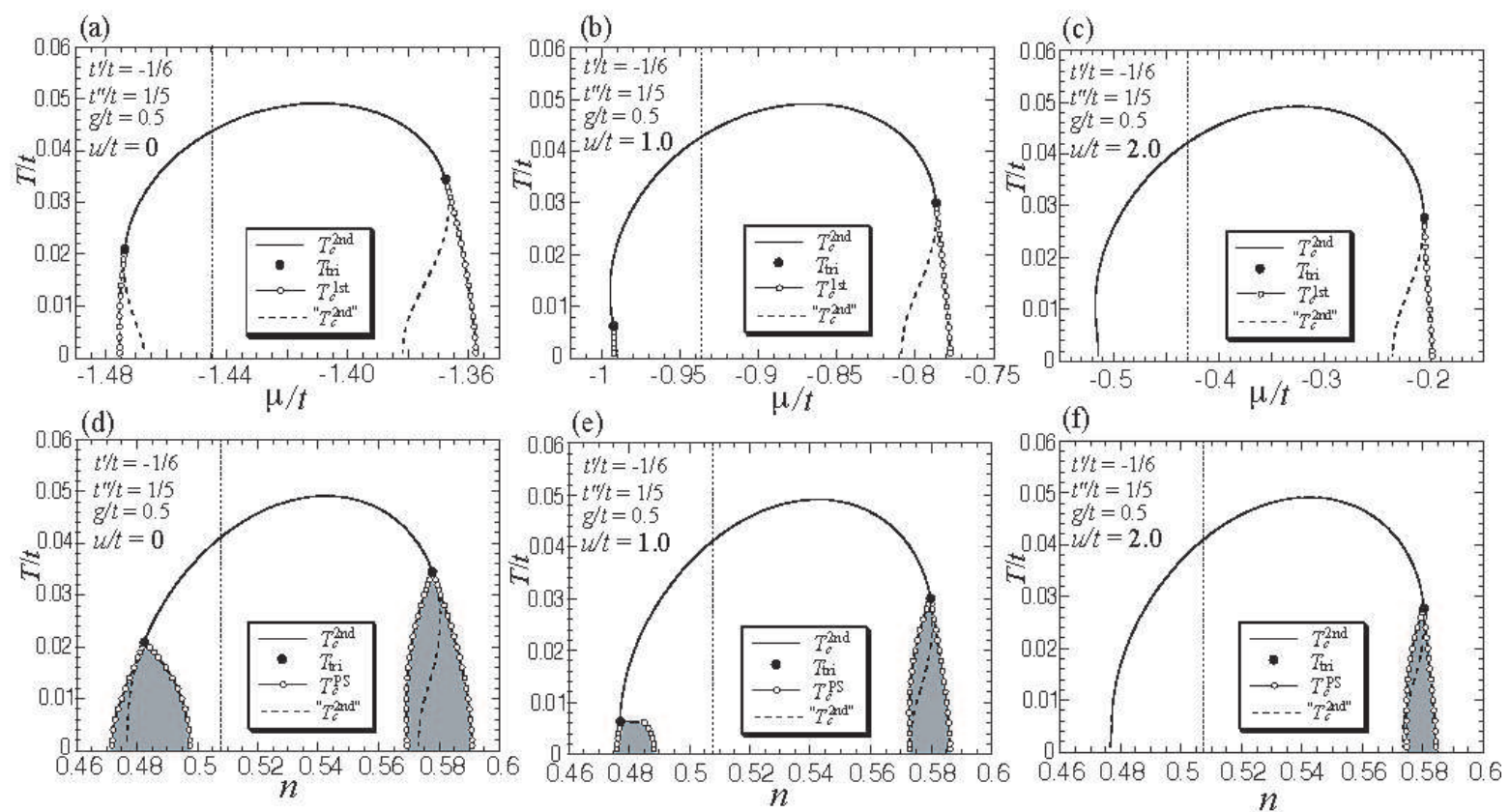

FIG. 4: $\mu$-T phase diagrams for several choices of $u$ for $t^{\prime} / t=-1 / 6, t^{\prime \prime} / t=1 / 5$, and $g / t=0.5$ : $u / t=0(\mathrm{a}), 1.0(\mathrm{~b})$, and 2.0 (c). The dotted lines indicate the van Hove energy. The lines of $T_{c}^{1 \text { st }}$ and " $T_{c}^{2 \text { nd " }}$ at lower $\mu$ in (b) are too close to be distinguished. The $\mu-T$ phase diagrams are replotted as a function of $n$ in (d)-(f).

$\alpha=\left(t+2 t^{\prime}\right) / 4 t^{\prime \prime}$, and local minima at $(\pi, 0)$ and $(0, \pi)$. The energy at the saddle points is given by $\epsilon_{\mathrm{vH}}^{0}=-2\left(t-2 \alpha^{2} t^{\prime \prime}\right)$. For $t^{\prime} / t=-1 / 6$ and $t^{\prime \prime} / t=1 / 5$ one has $\alpha=\frac{5}{6}$ and $\epsilon_{\mathrm{vH}}^{0}=-\frac{13}{9} t$.

A sequence of phase diagrams for $t^{\prime} / t=-1 / 6$ and $t^{\prime \prime} / t=1 / 5$ is plotted in Fig. 4 the coupling $g$ is $0.5 t$ in all cases. The transition line $T_{c}(\mu)$ for $u=0$ in Fig. \&(a) has a dome shape similar to that in Fig. 1(a), with a second order transition at high $T$ and a first order transition at low $T$. Although the Fermi surface symmetry breaking is mainly due to electrons close to the Fermi surface near the van Hove points, the maximum of $T_{c}(\mu)$ largely deviates from the van Hove energy and the dome shows a pronounced asymmetry. This is related to a large asymmetry of the density of states (see Sec. IV). A striking feature of the present parameters is a drastic suppression of one of the tricritical points by a moderate uniform repulsion in the interaction, which leads to a quantum critical point for sufficiently large $u$. We show $\mu-T$ phase diagrams for $u / t=1$ and 2 in Figs. 4(b) and (c), respectively. The tricritical point at higher $\mu$ is only slightly suppressed by $u$ and the suppression saturates 
at large $u$ as in the case of Fig. 2. However, the first order line at lower $\mu$ is suppressed rapidly with increasing $u$ and disappears completely in favor of a continuous phase transition down to the lowest temperatures beyond $u / t \sim 2$, that is a quantum critical point is realized. Why the choice of hopping parameters with saddle points deviating from $(\pi, 0)$ and $(0, \pi)$ is favorable for a continuous phase transition at low temperatures will be discussed in connection with the Landau expansion in Sec. IV.

In Figs. 4 (d)-(f), we replot the $\mu-T$ phase diagrams as a function of $n$. The first order transitions at low $T$ lead to phase separated regions as already seen in Fig. 1. However, on the lower density side of the $n$ - $T$ phase diagram in Fig. 4(e) the phase separated region opens discontinuously at the critical temperature $T_{\text {tri }}$, where the second order line terminates. In that case the end point of the second order line is not a tricritical point (and $T_{\text {tri }}$ thus actually a misnomer). The quartic term of the Landau energy is positive there, but a first order transition nevertheless sets in due to a local minimum at finite $\eta$ in the Landau energy becoming a global one below $T_{\text {tri }}$.

In Fig. [5] we show the $\mu$-dependence of the order parameter $\eta$ for hopping parameters and coupling $g$ as in Fig. 4, at a very low temperature $(T / t=0.001)$ and two different choices for $u$. This plot reveals that for $u / t=2$ there is a first order transition within the symmetry-broken phase in addition and very close to the quantum critical point shown in Fig. 4 (c). The corresponding Landau energy [Fig. 5] (b)] has two minima at finite $|\eta|$; the minimum at lower $|\eta|$ has the lowest energy only for $\mu$ between the second and first order transition point. For larger $u$ the first order transition in the symmetry-broken phase moves further away from the second order transition until it disappears completely. We show in Fig. 5 (c) that the order parameter for $u / t=10$ is continuous everywhere except at the first order transition at the large $\mu$ boundary of the symmetry-broken phase. The Landau energy for $\mu$ close to the second order transition has only one minimum as a function of $|\eta|$ in this case [Fig. [5 (d)].

No quantum critical point appears for $t^{\prime} / t=-1 / 6$ and $t^{\prime \prime} / t=1 / 5$ if the coupling constant $g$ is too small or too large. In Fig. 6] we plot $T_{\text {tri }}$ as a function of $u$ for $g / t=1.0$. Although $T_{\text {tri }}$ at lower $\mu$ is suppressed much stronger than that at higher $\mu$, the suppression saturates at a finite temperature even for large $u$. Remarkably the Stoner factor $S$ is again generally strongly enhanced for the present hopping parameters near the transition at higher $\mu$, while $S$ is not large at low $T$ near the transition at lower $\mu$ for $u=0$, although $T_{\text {tri }}$ is lower there. 

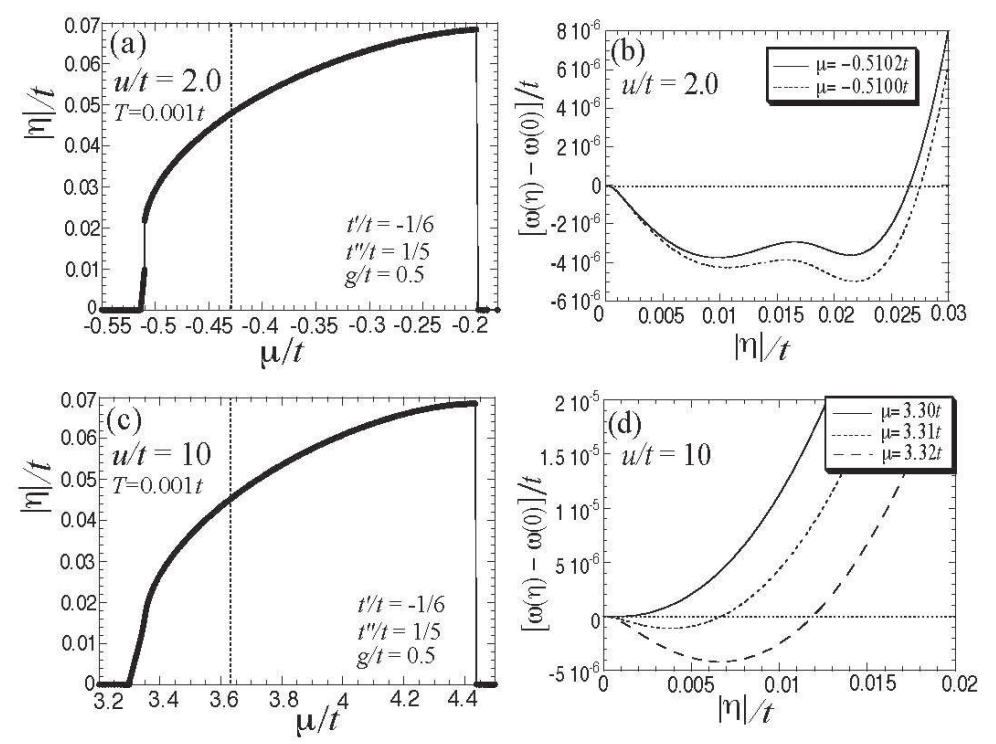

FIG. 5: Left panels: Order parameter $|\eta|$ as a function of $\mu$ for hopping parameters and $g$ as in Fig. 4. temperature $T / t=0.001$, and two different uniform couplings: $u / t=2$ (top) and $u / t=10$ (bottom). Right panels: Landau energy $\omega(\eta)$ near the phase transitions at the lower $\mu$ side of the corresponding order parameter plots on the left.

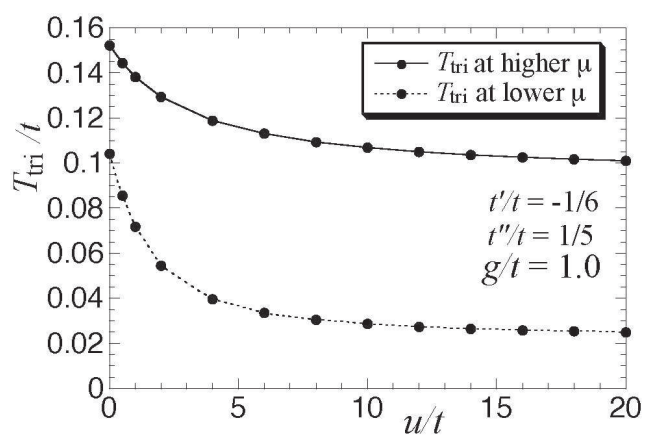

FIG. 6: $u$-dependence of $T_{\text {tri }}$ at higher $\mu$ (solid line) and at lower $\mu$ (dotted line) for $t^{\prime} / t=-1 / 6$, $t^{\prime \prime} / t=1 / 5$, and $g / t=1.0$.

Obviously a low $T_{\text {tri }}$ does not imply a large Stoner factor.

\section{LANDAU EXPANSION}

To gain a broader understanding of the symmetry-breaking phase transitions and their dependence on model parameters we consider the Landau expansion of the grand canonical 
potential in powers of the order parameter

$$
\omega(\eta)-\omega(0)=\frac{1}{2} a_{2} \eta^{2}+\frac{1}{4 !} a_{4} \eta^{4}+\ldots
$$

up to quartic order. The function $\omega(\eta)$ is given by Eq. (17), with the density $n(\eta)$ determined by Eq. (9). Note that only even powers appear due to the symmetry $\omega(-\eta)=\omega(\eta)$. Expanding $\omega(\eta)$ by taking $\eta$-derivatives (see Appendix) one obtains the coefficients

$$
\begin{aligned}
& a_{2}=g^{-1}-N_{2}(\bar{\mu}, T), \\
& a_{4}=-N_{4}^{\prime \prime}(\bar{\mu}, T)+\frac{3 u}{1+u N_{0}(\bar{\mu}, T)}\left[N_{2}^{\prime}(\bar{\mu}, T)\right]^{2},
\end{aligned}
$$

where $\bar{\mu}=\mu-u n(0)$ and

$$
N_{p}(\bar{\mu}, T)=-\frac{2}{L} \sum_{\mathbf{k}} d_{\mathbf{k}}^{p} f^{\prime}\left(\epsilon_{\mathbf{k}}^{0}-\bar{\mu}\right)
$$

is a weighted density of states averaged over an energy interval of order $T$ around $\bar{\mu}$, and $N_{p}^{\prime}$, $N_{p}^{\prime \prime}$ are first and second derivatives with respect to $\bar{\mu}$. Note that $a_{2}$ depends only via $\bar{\mu}$ on $u$, which explains the $u$-independence of the second order transition lines in the $(n, T)$ phase diagrams in Figs. 2 and 4. The quartic coefficient $a_{4}$ does not depend on $g$. In Fig. [7 we plot $N_{0}(\bar{\mu}, T), N_{2}^{\prime}(\bar{\mu}, T)$, and $N_{4}^{\prime \prime}(\bar{\mu}, T)$ for $T=0$ and $T=0.01 t$, for the choice of hoppings underlying the results in Sec. IIIA-C. At zero temperature $N_{p}(\bar{\mu}, T)$ diverges logarithmically for $\bar{\mu} \rightarrow \epsilon_{\mathrm{vH}}^{0}$, which leads to $N_{2}^{\prime}(\bar{\mu}, T) \propto-\left(\bar{\mu}-\epsilon_{\mathrm{vH}}^{0}\right)^{-1}$ and $N_{4}^{\prime \prime}(\bar{\mu}, T) \propto\left(\bar{\mu}-\epsilon_{\mathrm{vH}}^{0}\right)^{-2}$ near $\epsilon_{\mathrm{vH}}^{0}$. These singularities are cut off for $\left|\bar{\mu}-\epsilon_{\mathrm{vH}}^{0}\right|<T$ at finite temperature.

The critical manifold in the space spanned by $g, \bar{\mu}$, and $T$, on which a continuous phase transition may occur, is given by the condition $a_{2}=0$, that is $g N_{2}(\bar{\mu}, T)=1$. However, the continuous transition can be realized only if the quartic coefficient $a_{4}$ is positive. Otherwise it will be preempted by a first order transition. For $u=0$, one has simply $a_{4}=-N_{4}^{\prime \prime}(\bar{\mu}, T)$, which is obviously negative near the van Hove singularity at temperatures $T \ll\left|\bar{\mu}-\epsilon_{\mathrm{vH}}^{0}\right|$, and positive at any $T>0$ for $\bar{\mu}=\epsilon_{\mathrm{vH}}^{0}$. Hence, the transition is first order at temperatures $T \ll\left|\bar{\mu}-\epsilon_{\mathrm{vH}}^{0}\right|$, but it can be expected to be continuous near the maximum of $T_{c}$ around van Hove filling, as is indeed the case in all numerical results. For $u>0$ there is an additional positive contribution to $a_{4}$, which partially compensates the negative main term. However, the positive term is bounded by $3\left[N_{2}^{\prime}(\bar{\mu}, T)\right]^{2} / N_{0}(\bar{\mu}, T)$ even for arbitrarily large $u$. For $\bar{\mu}$ near $\epsilon_{\mathrm{vH}}^{0}$ it is of order $1 / \log \left|\bar{\mu}-\epsilon_{\mathrm{vH}}^{0}\right|$ smaller than $N_{4}^{\prime \prime}(\bar{\mu}, T)$. 

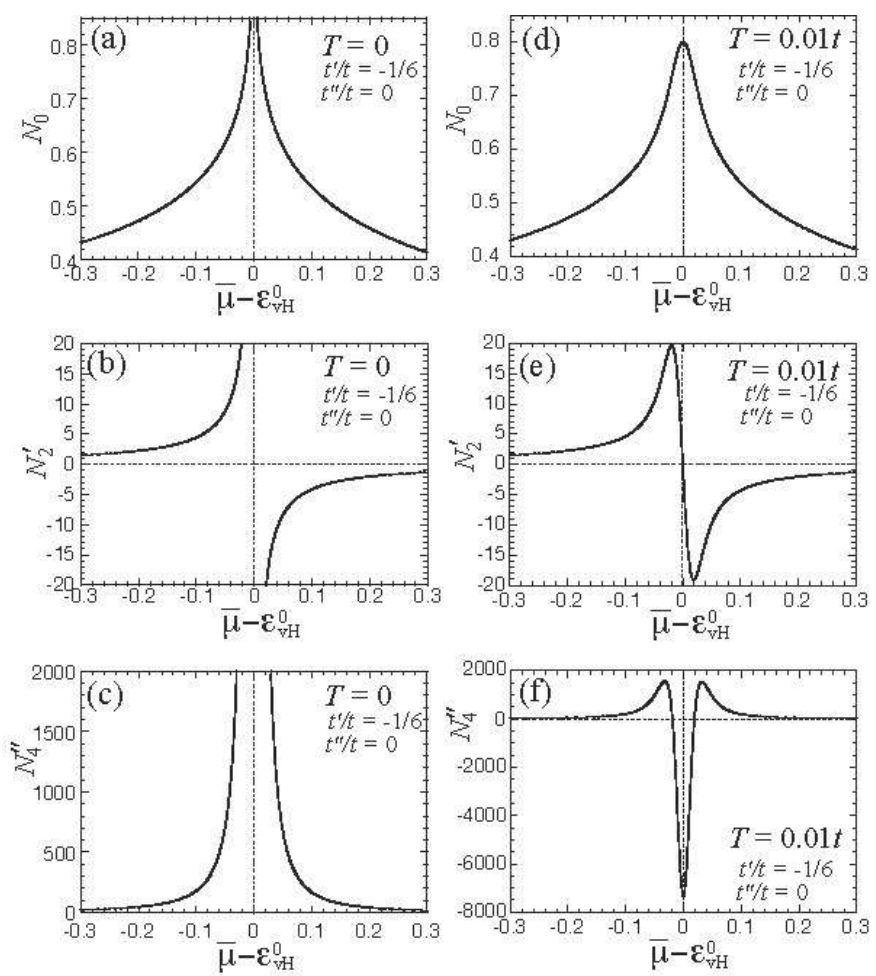

FIG. 7: The functions $N_{0}(\bar{\mu}, T), N_{2}^{\prime}(\bar{\mu}, T)$, and $N_{4}^{\prime \prime}(\bar{\mu}, T)$ around the van Hove energy at $T=0$ [(a)-(c)] and $T=0.01 t[(\mathrm{~d})-(\mathrm{f})]$ for hopping parameters $t^{\prime} / t=-1 / 6$ and $t^{\prime \prime}=0$; the energy unit is $t$.

At moderate distance from van Hove filling a numerical evaluation reveals that for hopping parameters $t^{\prime} / t=-1 / 6$ and $t^{\prime \prime}=0$ the positive $u$-term can compensate a substantial amount of the negative term, $-N_{4}^{\prime \prime}(\bar{\mu}, T)$, but $a_{4}$ never turns positive. We thus confirm that a finite $u$ shifts the tricritical points in the phase diagrams to lower temperatures, but does not produce a continuous phase transition at $T=0$ for that choice of hopping.

The situation is very different for our second choice, $t^{\prime} / t=-1 / 6$ and $t^{\prime \prime} / t=1 / 5$. In that case $N_{p}(\bar{\mu}, T)$ has a step-like increase at the lower $\mu$ side of the van Hove energy at low $T$, which is generated by the local minima in the dispersion at $(\pi, 0)$ and $(0, \pi)$. This is illustrated for $N_{0}$ in Fig. [8, $N_{2}$ and $N_{4}$ behave similarly. For $\bar{\mu}$ in the step region $N_{2}^{\prime}(\bar{\mu}, T)$ becomes very large at low $T$, while $N_{0}(\bar{\mu}, T)$ remains bounded. In the presence of a $u$-term it is thus possible to get a positive $a_{4}$ at arbitrarily low temperatures. A sizable $u$ helps not only to increase $a_{4}$, but also to push local minima at finite $\eta$ in $\omega(\eta)$ to higher energies, such that a continuous transition can be obtained before such a minimum becomes global. 

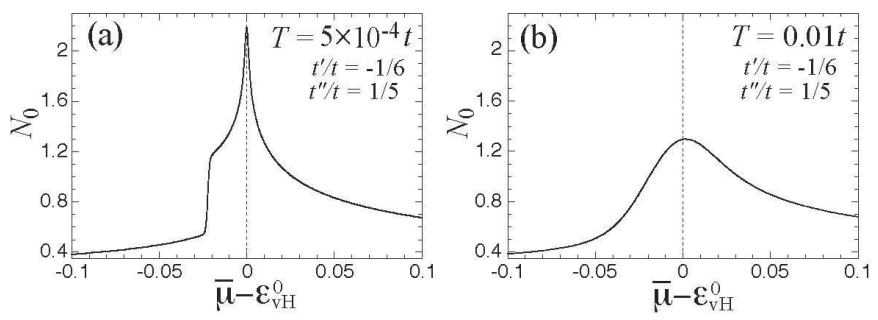

FIG. 8: $N_{0}(\bar{\mu}, T)$ around the van Hove energy at $T=5 \times 10^{-4} t$ (a) and $T=0.01 t$ (b) for hopping parameters $t^{\prime} / t=-1 / 6$ and $t^{\prime \prime}=1 / 5$; the energy unit is $t$.

\section{WEAK COUPLING LIMIT}

At weak coupling spontaneous Fermi surface symmetry breaking occurs only for densities near van Hove filling, and the transition is dominated by states with momenta near the saddle points of $\epsilon_{\mathbf{k}}^{0}$. In this limit the mean-field equations can be treated to a certain extent analytically, and the phase transition is universal in the sense that it is fully characterized by a single energy scale. Several ratios of physical quantities are universal dimensionless numbers in the weak coupling limit.

We focus on the case $u=0$ and assume that $\epsilon_{\mathbf{k}}^{0}$ has only two degenerate saddle points in $\mathbf{k}_{A}=(\pi, 0)$ and $\mathbf{k}_{B}=(0, \pi)$. However, the following analysis can be easily extended to energy bands with saddle points in other positions. Shifting energies such that $\epsilon_{\mathbf{k}_{A, B}}^{0}=0$ and choosing suitable relative momentum variables $k_{+}$and $k_{-}$, one can write $\epsilon_{\mathbf{k}}^{0}$ near the saddle points as a quadratic form

$$
\epsilon_{\mathbf{k}}^{0}=\frac{1}{2 m} k_{+} k_{-},
$$

where $m>0$ is a constant which can be related to the hopping amplitudes $t, t^{\prime}$, and $t^{\prime \prime}$. The variables $k_{+}$and $k_{-}$are defined such that the quadratic form has no $k_{+}^{2}$ and $k_{-}^{2}$ terms and that the integration measure is $(2 \pi)^{-2}$ as usual. Note that the corresponding coordinate axes are generally not orthogonal. The momenta are restricted to a finite region around the saddle points by a cutoff $\Lambda$, that is $\left|k_{ \pm}\right| \leq \Lambda$.

If the form factor $d_{\mathbf{k}}$ is smooth near the van Hove point it can be taken as a constant with alternating sign near $\mathbf{k}_{A}$ and $\mathbf{k}_{B}$, respectively, such as

$$
d_{\mathbf{k}}=\left\{\begin{array}{r}
1 \text { for } \mathbf{k} \approx \mathbf{k}_{A} \\
-1 \text { for } \mathbf{k} \approx \mathbf{k}_{B}
\end{array}\right.
$$


Any other constant $(\neq 1)$ could be absorbed in the coupling $g$. The self-consistency condition Eq. (8) becomes simply

$$
\eta=g\left(n_{B}-n_{A}\right)
$$

where

$$
n_{A, B}=2 \int_{A, B} \frac{d^{2} k}{(2 \pi)^{2}} f\left(\epsilon_{\mathbf{k}}^{0} \pm \eta-\mu\right)
$$

is the contribution of the momentum space region around $\mathbf{k}_{A}$ and $\mathbf{k}_{B}$ (limited by $\Lambda$ ) to the density.

\section{A. Ground state}

At $T=0$ the integral over the Fermi function is simply the volume of occupied states. An elementary integration yields

$$
n_{A, B}=\frac{\Lambda^{2}}{\pi^{2}}+\frac{2 m}{\pi^{2}}(\mu \mp \eta)\left(1+\log \frac{\Lambda^{2}}{2 m}-\log |\mu \mp \eta|\right) .
$$

The self-consistency equation can thus be written as

$$
\eta=\bar{g}\left[(\mu-\eta) \log |\mu-\eta|-(\mu+\eta) \log |\mu+\eta|+2 \eta\left(1+\log \epsilon_{\Lambda}\right)\right]
$$

with the dimensionless coupling $\bar{g}=\frac{2 m}{\pi^{2}} g$ and the cutoff energy $\epsilon_{\Lambda}=\Lambda^{2} / 2 m$. Integrating $\partial \omega / \partial \eta=g^{-1} \eta-\left(n_{B}-n_{A}\right)$ over $\eta$ one obtains the $\eta$-dependence of the grand canonical potential

$$
\begin{aligned}
\omega(\eta)= & \frac{2 m}{\pi^{2}}\left\{\left[\frac{1}{2 \bar{g}}-\frac{1}{2}-\left(1+\log \epsilon_{\Lambda}\right)\right] \eta^{2}\right. \\
& \left.+\frac{1}{2}(\mu+\eta)^{2} \log |\mu+\eta|+\frac{1}{2}(\mu-\eta)^{2} \log |\mu-\eta|\right\}+ \text { const. }
\end{aligned}
$$

where the constant does not depend on $\eta$ and can be chosen such that $\omega(0)=0$.

For $\mu=0$, where the bare Fermi surface touches the van Hove points, the self-consistency equation for $\eta$ becomes simply

$$
\eta=-2 \bar{g} \eta \log |\eta|+2 \bar{g} \eta \log \left(e \epsilon_{\Lambda}\right)
$$

Besides the trivial solution $\eta=0$ this equation has the two degenerate solutions

$$
\eta_{0}= \pm e \epsilon_{\Lambda} e^{-1 /(2 \bar{g})}
$$


It is easy to see that the solution $\eta=0$ corresponds to a maximum of $\omega(\eta)$ and is thus unstable. The total density remains unaffected by the symmetry breaking, since $\delta n_{B}=-\delta n_{A}$ for $\mu=0$. This is also true to high accuracy for the density at van Hove filling for the numerical result shown in Fig. 1(e). Note that at van Hove filling symmetry breaking occurs for arbitrarily small $g$, which is due to the logarithmic divergence of the density of states.

For $\mu \neq 0$ we introduce a rescaled order parameter $\tilde{\eta}=\eta / \mu$. The self-consistency condition for $\eta$ can be written in terms of $\tilde{\eta}$ as

$$
\frac{\tilde{\eta}}{\tilde{g}}=(1-\tilde{\eta}) \log |1-\tilde{\eta}|-(1+\tilde{\eta}) \log |1+\tilde{\eta}|
$$

with a renormalized coupling constant given by

$$
\frac{1}{\tilde{g}}=\frac{1}{\bar{g}}+2 \log |\mu|-2\left(1+\log \epsilon_{\Lambda}\right) .
$$

The grand canonical potential can be written as $\omega(\eta)=\frac{2 m}{\pi^{2}} \mu^{2} \tilde{\omega}(\tilde{\eta})$, where

$$
\tilde{\omega}(\tilde{\eta})=\left(\frac{1}{2 \tilde{g}}-\frac{1}{2}\right) \tilde{\eta}^{2}+\frac{1}{2}(1+\tilde{\eta})^{2} \log |1+\tilde{\eta}|+\frac{1}{2}(1-\tilde{\eta})^{2} \log |1-\tilde{\eta}| .
$$

Note that $\mu$ and the cutoff have been completely absorbed in the renormalized coupling $\tilde{g}$. The rescaled self-consistency equation and $\tilde{\omega}(\tilde{\eta})$ are universal in the sense that they depend only via $\tilde{g}$ on all input parameters.

Minimizing $\tilde{\omega}(\tilde{\eta})$ one finds that a first order transition occurs at the universal critical coupling $\tilde{g}_{1} \approx-0.692$ with a universal jump of the dimensionless order parameter

$$
\left|\tilde{\eta}_{1}\right|=\frac{\left|\eta_{1}\right|}{\left|\mu_{1}\right|} \approx 1.720
$$

The Fermi surface thus opens immediately at the transition. Inverting the relation between $\bar{g}$ and $\tilde{g}$ one obtains the $\mu$-dependence of $\bar{g}_{1}$ as

$$
\bar{g}_{1}(\mu)=\frac{\tilde{g}_{1}}{1+2 \tilde{g}_{1}\left(\log \frac{\epsilon_{\Lambda}}{|\mu|}+1\right)} \quad \stackrel{\mu \rightarrow 0}{\rightarrow} \frac{1}{2 \log \frac{\epsilon_{\Lambda}}{|\mu|}} .
$$

Note that $\bar{g}_{1}>0$ for $|\mu| \ll \epsilon_{\Lambda}$ although $\tilde{g}_{1}<0$. For fixed $g$, on the other hand, the critical value of $\mu$ at which the first order transition occurs is

$$
\left|\mu_{1}\right|=e^{1+1 /\left(2 \tilde{g}_{1}\right)} \epsilon_{\Lambda} e^{-1 /(2 \bar{g})} .
$$

The curvature of $\tilde{\omega}(\tilde{\eta})$ at $\tilde{\eta}=0$ becomes negative only for $\tilde{g}>\tilde{g}_{2}=-0.5$. At fixed $\mu$ this requires couplings $g>g_{2}(\mu)>g_{1}(\mu)$. For given $g$, the critical value of $\mu$ for a continuous transition is

$$
\left|\mu_{2}\right|=\epsilon_{\Lambda} e^{-1 /(2 \bar{g})}
$$


which is smaller than $\left|\mu_{1}\right|$. Hence, the critical point for a continuous transition is not reached, but preempted by a first order transition. The ratio $\left|\mu_{1}\right| /\left|\mu_{2}\right|=e^{1+1 /\left(2 \tilde{g}_{1}\right)} \approx 1.320$ is a universal number.

In the symmetric state, the interaction induced enhancement of the $d$-wave compressibility of the Fermi surface is given by the "Stoner factor" $S=\left[1-g N_{2}(\mu)\right]^{-1}$, which is related to the quadratic coefficient of the Landau expansion by $S=\left(g a_{2}\right)^{-1}$, see Eq. (14). Since $a_{2}$ vanishes for $g=g_{2}$, one has $a_{2}=g^{-1}-g_{2}^{-1}$. At the first order transition in the ground state, $a_{2}$ is given by

$$
\left.a_{2}\right|_{1}=g_{1}^{-1}-g_{2}^{-1}=\frac{2 m}{\pi^{2}}\left(\tilde{g}_{1}^{-1}-\tilde{g}_{2}^{-1}\right) \approx 0.555 \frac{2 m}{\pi^{2}},
$$

where we have used Eq. (27) in the second step. The $d$-wave compressibility is thus enhanced by a factor

$$
S_{1}=\frac{1}{0.555 \bar{g}}
$$

at the first order transition. For a weak coupling $\bar{g} \ll 1$, this enhancement is very large.

\section{B. Finite temperature}

We now compute two characteric temperature scales in the weak coupling limit, namely the transition temperature at van Hove filling, $T_{0}$, and the tricritical temperature, $T_{\text {tri }}$. To this end we write the functions $N_{p}(\mu, T)$ in the form

$$
N_{p}(\mu, T)=-\int d \epsilon N_{p}(\epsilon) f^{\prime}(\epsilon-\mu)
$$

where $N_{p}(\epsilon)=N_{p}(\epsilon, 0)$, which, for the quadratic dispersion in Eq. (17) with a cutoff $\Lambda$, is given by

$$
N_{p}(\epsilon)=\frac{4 m}{\pi^{2}} \log \frac{\epsilon_{\Lambda}}{|\epsilon|}
$$

with $|\epsilon| \leq \epsilon_{\Lambda}$. Note that $\bar{\mu}=\mu$ for $u=0$.

The transition temperature at van Hove filling is obtained by setting the quadratic coefficient in the Landau expansion $a_{2}$ to zero at $\mu=0$, that is by solving the equation $g N_{2}\left(0, T_{0}\right)=1$. Using

$$
\int_{-\epsilon_{\Lambda}}^{\epsilon_{\Lambda}} d \epsilon \log \frac{|\epsilon|}{\epsilon_{\Lambda}} f^{\prime}(\epsilon) \stackrel{\epsilon_{\Lambda} / T \rightarrow \infty}{\longrightarrow} \log \frac{\epsilon_{\Lambda}}{T}-\log \frac{\pi}{2}+\gamma
$$


where $\gamma \approx 0.577$ is the Euler constant, one obtains

$$
T_{0}=\frac{2 e^{\gamma}}{\pi} \epsilon_{\Lambda} e^{-1 /(2 \bar{g})}
$$

Note that the numerical prefactor coincides precisely with the one in the BCS formula for the critical temperature of a weak coupling superconductor.

At the tricritical point $a_{2}$ and $a_{4}$ both vanish. The tricritical temperature $T_{\text {tri }}$ and the corresponding chemical potential $\mu_{\text {tri }}$ are thus determined by the two equations $g N_{2}\left(\mu_{\text {tri }}, T_{\text {tri }}\right)=$ 1 and $N_{4}^{\prime \prime}\left(\mu_{\text {tri }}, T_{\text {tri }}\right)=0$. Using Eqs. (35) and (36) one obtains

$$
g N_{2}(\mu, T) \stackrel{\epsilon_{\Lambda} / T \rightarrow \infty}{\longrightarrow} 2 \bar{g}\left[\log \frac{\epsilon_{\Lambda}}{T}+a(\tilde{\mu})\right]
$$

where $\tilde{\mu}=\mu / T$ and the dimensionless function $a(\tilde{\mu})$ is defined as

$$
a(\tilde{\mu})=\int_{-\infty}^{\infty} d x \log |x+\tilde{\mu}| \frac{\partial}{\partial x} \frac{1}{e^{x}+1} .
$$

Furthermore

$$
N_{4}^{\prime \prime}(\mu, T) \stackrel{\epsilon_{\Lambda} / T \rightarrow \infty}{\longrightarrow} \frac{4 m}{(\pi T)^{2}} b(\tilde{\mu})
$$

with

$$
b(\tilde{\mu})=\int_{-\infty}^{\infty} d x \log |x+\tilde{\mu}| \frac{\partial^{3}}{\partial x^{3}} \frac{1}{e^{x}+1} .
$$

The latter function vanishes for $\tilde{\mu}= \pm \tilde{\mu}_{\text {tri }}$ with $\tilde{\mu}_{\text {tri }} \approx 1.911$. Setting the right hand side of Eq. (39) equal to one and solving for $T$ then yields

$$
T_{\text {tri }}=e^{-\alpha} \epsilon_{\Lambda} e^{-1 /(2 \bar{g})}
$$

where $\alpha=a\left(\tilde{\mu}_{\text {tri }}\right) \approx 0.4515$. Hence, the tricritical temperature and the critical temperature at van Hove filling form the universal ratio

$$
\frac{T_{\text {tri }}}{T_{0}}=\frac{\pi e^{-\alpha}}{2 e^{\gamma}} \approx 0.5614
$$

\section{Comparison with numerical results}

Above we have computed several physical quantities characterizing the phase transition, which are all proportional to the same energy scale $\epsilon_{\Lambda} e^{-1 /(2 \bar{g})}$, with universal prefactors in the weak coupling limit. Hence ratios of these quantities are universal numbers. 
We have checked universal ratios against results from the numerical solution of the meanfield equations for hopping parameters $t^{\prime} / t=-1 / 6, t^{\prime \prime}=0$ and coupling $u=0$. At zero temperature we have checked ratios involving the order parameter $\eta_{0}$ at van Hove filling, the order parameter jump $\eta_{1}$ at the first order transition, and the distances of $\mu_{1}$ and $\mu_{2}$ from $\epsilon_{\mathrm{vH}}^{0}$. For a comparison of the order parameter one has to take into account a factor two due to the different size of $d_{\mathbf{k}}$ near the van Hove points, which is \pm 2 in the numerical calculation with $d_{\mathbf{k}}=\cos k_{x}-\cos k_{y}$, but \pm 1 in the weak coupling model. At finite temperature we have compared the transition temperature $T_{0}$ at van Hove filling and the tricritical point. For $g / t=0.5$ all ratios agree within one percent error with the predicted universal numbers. For the stronger coupling $g / t=1$ the deviation increases to two or three percent at zero temperature and up to around five percent at finite temperature.

\section{CONCLUSION}

In summary, we have analyzed a mean-field model for Fermi surface symmetry breaking with a $d$-wave order parameter on a square lattice. We have confirmed the qualitative

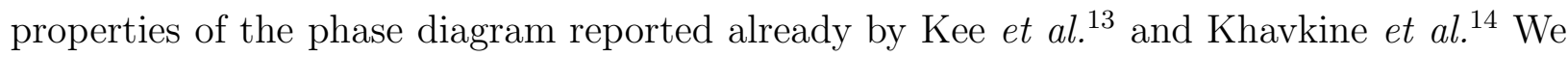
have provided further numerical evidence and analytic arguments showing that the phase transition is typically first order at low temperatures. This implies that a stability analysis of microscopic models, for example by renormalization group methods, should not focus on diverging susceptibilities only. At weak coupling the transition is fully characterized by a single energy scale and can thus be described by universal dimensionless functions of suitably rescaled parameters, which leads to various universal ratios of different quantities. The tricritical points separating first and second order behavior are shifted to lower temperatures

by adding a repulsive constant contribution to the forward scattering interaction, which, for a favorable choice of hopping and interaction parameters, can even lead to a quantum critical point. Although the phase transition is usually first order at low $T$, we have found that the $d$-wave compressibility at the transition can be enhanced significantly by interactions, which implies that Fermi surface fluctuations induced by interactions with a small finite momentum transfer are expected to be important even near the first order transition. The role of fluctuations, in particular their influence on the phase transition, remains an interesting subject for future studies. 


\section{Acknowledgments}

We are grateful to L. Dell'Anna for valuable discussions. V.O. thanks I. Khavkine, C. Chung and H. Kee for collaboration on a related work, and NSF-DMR-0213706 and the David and Lucile Packard Foundation grants for support. H.Y. has been partially supported by a special postdoctoral researchers program from RIKEN, Japan.

\section{APPENDIX A: DERIVATION OF LANDAU EXPANSION}

Here we derive the expressions Eqs. (14) and (15) for the coefficients of the Landau expansion of $\omega(\eta)$, by taking derivatives with respect to $\eta$ at fixed $\mu$ and with $n(\eta)$ determined by Eq. (91). The first derivative is

$$
\frac{d \omega}{d \eta}=\frac{\partial \omega}{\partial \eta}+\frac{\partial \omega}{\partial n} \frac{d n}{d \eta}=\frac{\eta}{g}+\frac{2}{L} \sum_{\mathbf{k}} d_{\mathbf{k}} f\left(\epsilon_{\mathbf{k}}-\mu\right)
$$

where we have used the stationarity condition $\frac{\partial \omega}{\partial n}=0$. The second derivative is

$$
\frac{d^{2} \omega}{d \eta^{2}}=g^{-1}+\frac{2}{L} \sum_{\mathbf{k}}\left[d_{\mathbf{k}}^{2}+d_{\mathbf{k}} u \frac{d n}{d \eta}\right] f^{\prime}\left(\epsilon_{\mathbf{k}}-\mu\right) .
$$

Exploiting the symmetry $n(\eta)=n(-\eta)$, which implies that odd derivatives of $n(\eta)$ vanish at $\eta=0$, one obtains

$$
a_{2}=\left.\frac{d^{2} \omega}{d \eta^{2}}\right|_{\eta=0}=g^{-1}+\frac{2}{L} \sum_{\mathbf{k}} d_{\mathbf{k}}^{2} f^{\prime}\left(\epsilon_{\mathbf{k}}^{0}+u n(0)-\mu\right)=g^{-1}-N_{2}(\bar{\mu}, T)
$$

with $\bar{\mu}=\mu-u n(0)$.

Differentiating twice more and setting $\eta=0$, one obtains

$$
a_{4}=\left.\frac{d^{4} \omega}{d \eta^{4}}\right|_{\eta=0}=-N_{4}^{\prime \prime}(\bar{\mu}, T)+\left.3 u N_{2}^{\prime}(\bar{\mu}, T) \frac{d^{2} n}{d \eta^{2}}\right|_{\eta=0}
$$

Applying two $\eta$-derivatives to $n(\eta)$ as given by Eq. (9) we get

$$
\left.\frac{d^{2} n}{d \eta^{2}}\right|_{\eta=0}=N_{2}^{\prime}(\bar{\mu}, T)-\left.u N_{0}(\bar{\mu}, T) \frac{d^{2} n}{d \eta^{2}}\right|_{\eta=0} .
$$

Solving for $n^{\prime \prime}(0)$ and inserting into Eq. (A4) we obtain Eq. (15).

1 H. Yamase and H. Kohno, J. Phys. Soc. Jpn. 69, 332 (2000); 69, 2151 (2000). 
2 C.J. Halboth and W. Metzner, Phys. Rev. Lett. 85, 5162 (2000).

3 I. Grote, E. Körding, and F. Wegner, J. Low Temp. Phys. 126, 1385 (2002); V. Hankevych, I. Grote, and F. Wegner, Phys. Rev. B 66, 094516 (2002).

4 A. Neumayr and W. Metzner, Phys. Rev. B 67, 035112 (2003).

5 B. Valenzuela and M.A.H. Vozmediano, Phys. Rev. B 63, 153103 (2001).

6 I.J. Pomeranchuk, Sov. Phys. JETP 8, 361 (1958).

7 V. Oganesyan, S.A. Kivelson, and E. Fradkin, Phys. Rev. B 64, 195109 (2001).

8 S.A. Kivelson, E. Fradkin, and V.J. Emery, Nature 393, 550 (1998).

9 Possible experimental signatures of fluctuating charge order in cuprate superconductors are discussed by S.A. Kivelson, I.P. Bindloss, E. Fradkin, V. Oganesyan, J.M. Tranquada, A. Kapitulnik, and C. Howald, Rev. Mod. Phys. 75, 1201 (2003).

10 H. Yamase, Phys. Rev. Lett. 93, 266404 (2004).

11 H. Yamase and H. Kohno, J. Phys. Soc. Jpn. 70, 2733 (2001); H. Yamase, J. Phys. Soc. Jpn. 71, 1154 (2002); H. Yamase and H. Kohno, Phys. Rev. B 68, 014502 (2003).

12 W. Metzner, D. Rohe, and S. Andergassen, Phys. Rev. Lett. 91, 066402 (2003).

13 H.-Y. Kee, E.H. Kim, and C.-H. Chung, Phys. Rev. B 68, 245109 (2003).

14 I. Khavkine, C.-H. Chung, V. Oganesyan, and H.-Y. Kee, Phys. Rev. B 70, 155110 (2004). 